\title{
Excesos de la metodolatría en la investigación supuestamente científica
}

\author{
Dr. Víctor Hugo Martel Vidal \\ Universidad Nacional de Educación Enrique Guzmán y Valle
}

A Mary Daly: quien padeció bajo el poder de la necia metodólatras y hace poco se libró de ellos para siempre

\section{Resumen}

La mayor parte de las investigaciones que se realizan en nuestra comunidad académica, se encuentran orientadas por la excluyente influencia de los métodos que se emplean, omitiendo la pertinencia epistemológica de los problemas que se proponen estudiar. Esta tendencia abrumadora solo ha conseguido caricaturizar a muchas de tales investigaciones, las que no pueden ocultar su trivialidad e inconsistencia, en cuanto a caricatura. En estos casos la metodología es utilizada como un cosmético que no ha logrado ocultar sus falencias.

Palabras clave: Metodolatría, pertinencia epistemológica, caricatura, cosmético.

\section{Methodolatry excess of alleged scientific research}

\begin{abstract}
Most of the research being conducted in our academic community, they are guided by the exclusive influence of used methods, omitting the epistemological relevance of the problems we intend to explore. This overwhelming trend has only gained to caricature many of these investigations; they can not hide its triviality and inconsistency, like a cartoon. In these cases the methodology has used like a cosmetic that it has not hidden their shortcomings.
\end{abstract}

Key words: Methodolatry, epistemological relevance, cartoon, cosmetic.

\section{¿En qué consiste la metodolatría?}

El término metodolatría fue acuñado por Mary Daly en 1973 en “Mas allá de Dios Padre, una filosofía hacia la liberación de las mujeres" y más tarde se ha definido como la forma más común del dogmatismo académico (Feyerabend, 2006): como la glorificación del método como si se tratara de un Dios, el conocimiento encasillado en constructos prefabricados, teniendo lazos de conectividad con nuevos descubrimientos, previniendo la resolución de nuevas preguntas, a través de la eliminación de ideas que no son responsables de las categorías de cuestionamiento y respuestas. La Dra. Daly, (1928-2010) filósofa norteamericana, ejerció la docencia en el Boston Collage desde 1967 hasta 1999.

Se denomina como metodólatras a quienes rinden culto dogmático e ingenuo, la pasión, adoración y esclavitud al uso de los métodos sobre todo los cuantitativos, como único requisito de cientificidad en la investigación que suponemos como "científica", sin considerar la pertinencia de los problemas que se hayan propuesto y mucho menos de los respectivos sistemas de hipótesis que comportan. Musso (1970) considera que para conseguir tal pertinencia se requiere de un supuesto teórico lo suficientemente consistente como para explicar minuciosamente el problema propuesto que contenga las variables a probar y sus respectivos indicadores.

Esta actividad corresponde a la epistemología, cuya tarea es la de estimar la validez de las teorías que suponemos científicas, que permitan encontrar la información y los conocimientos que se requieren para el planteamiento de los problemas de investigación y su correspondiente sistema de hipótesis donde se pongan a prueba las variables que comportan los problemas planteados. Recién en estas condiciones será posible elegir los métodos más apropiados para su estudio, ya que todo problema se anuncia en un primer momento como una simple sospecha dentro del sentido común y en esta condición es imposible empezar a investigar rigurosamente. En este estado lo recomendable es el primer nivel de la investigación: el exploratorio. 
Sin embargo, más de las veces, muchos investigadores, sobre todo los eventuales, que lo hacen debido a una exigencia académica para su graduación, saltan de este primer nivel de la investigación exploratoria y la convierten en una "investigación descriptiva" e incluso en una "explicativa" alentados por metodólogos dogmáticos e ingenuos refractarios a la reflexión teórica, filosófica y, mucho más epistemológica.

\section{Niveles de investigación científica}

\section{Primer nivel: investigación exploratoria}

Intenta responder a la interrogante ¿Qué es el problema que nos convoca? Si tomamos como ejemplo nuestro interés por Los Problemas en el Aprendizaje, luego de un esfuerzo acopiando información, casuística y bibliográfica podemos convenir con Condemarín (1969)en señalar que nos encontramos frente a un problema en el aprendizaje cuando un alumno no logra alcanzar los objetivos propuestos en un plazo determinado: comprensión lectora, cálculo lógico matemático, etc.

Esto significa que, frente a otros casos similares, podemos afirmar que nos encontramos frente a un problema en el aprendizaje, aunque no conozcamos su naturaleza; es decir, a pesar de que no sepamos a qué factores se encuentran asociados a ella, si existe algún componente orgánico o si se trata de otro tipo de carencias, propias de una sociedad tan heterogénea como la nuestra. En este nivel ya podemos elaborar cierto tipo de conjeturas, que luego de probarlas podemos confirmarlas o rechazarlas en los niveles subsiguientes.

Muchas veces a partir de este estado inicial y sin tener una información suficiente, que les permita definir en mejores condiciones el problema a investigar, las variables que intervienen, sus indicadores, con sus respectivos sistemas de hipótesis, sin conocer el supuesto teórico que les oriente y recurriendo a unas pocas teorías fragmentarias pre-científicas sobre el comportamiento humano, recurriendo a expertos en el empleo de métodos estadísticos se "convierten" en investigaciones de fachada científica. Esto es lo que avinagra, con frecuencia, las disputas académicas en la docencia universitaria (Eco, 1989).

\section{Segundo nivel: investigación descriptiva}

Aquí se intenta responder a la interrogante ¿Cómo es el problema que nos interesa estudiar? En este nivel podemos encontrar una serie de características propias de los problemas en el aprendizaje, entre los que destacan la falta de atención en el alumno, "se distrae fácilmente". "No puede mantener la atención". "Se fatiga prematuramente". Etc., Estas características se asocian también a la pertenencia a los grupos de riesgo, y a la evasión de las responsabilidades académicas. "No cumple con las tareas escolares". "Muestra descuido por su apariencia personal". etc.

De acuerdo con Sánchez (2008), a los factores mencionados podemos agregar otros factores socio-económicos, estados de marginalidad, familias disfuncionales, maltrato, abandono material, contextos sociales que lo exponen a comportamientos antisociales, el pandillaje, la inseguridad urbana, tugurización, promiscuidad, etc. Algunas de estas características pueden estar asociadas al problema que nos convoca, probablemente unas más que otras. El empleo de los instrumentos de medición puede ayudarnos a averiguar el impacto de estas características y cómo se asocian. Para averiguar qué características intervienen más que otras requerimos acceder al siguiente nivel de investigación.

En este nivel ya se cuenta con el supuesto teórico requerido para orientar gran parte del proceso, se puede elaborar ya el problema de investigación, reconocer las variables que intervienen, con sus correspondientes indicadores. En estas condiciones, siguiendo a Eco (1986) y a Hernández (2003) se pueden formular mejor el sistema de hipótesis y recurrir al empleo de los métodos más adecuados para comprobarlas. 


\section{Tercer nivel: investigación explicativa}

Intenta responder a la interrogante ¿Por qué este problema se conduce de una manera particular en cada caso y no de otra? Siguiendo con el ejemplo anterior, es probable que los problemas en el aprendizaje se encuentren no solo asociados, con frecuencia condicionados a la falta de madurez en los niños desde el inicio de su escolaridad (Encinas 1938, Condemarín, 1996) ocasionados por la disfuncionalidad entre los miembros de la familia que frecuentemente es anterior a la llegada de los niños (Rotondo, 1987).

Una de las características de tal disfuncionalidad se evidencia en la falta de tolerancia entre sus miembros (Rotondo, 1987), cuando se comprueba que muchas familias se encuentran en un estado de precariedad como consecuencia de esta falta de tolerancia que, a su vez es un indicador de falta de madurez cognitiva y afectiva, donde padres precoces crían hijos precoces (Condemarín, 1996). Aquí la precocidad se entiende como el inicio de la paternidad prematura y el inicio de la escolaridad, igualmente prematura, por carecer de la madurez cognitiva necesaria para reconocer la pertinencia de ambas, tanto la escolaridad en los niños, como la paternidad en los adultos. Ambos terminan improvisando sus roles, para luego evadirlos.

Aquí hay que señalar igualmente que la mayor parte de estos problemas no son resueltos adecuadamente, muchos persisten hasta cuando el niño llega a la adultez y nuevamente, les imponen sus problemas no resueltos a sus hijos. En la actividad laboral se observa lo mismo. Como ocurre en diversas instituciones no solo públicas, las privadas también muestran evidentes deficiencias, tanto de servicios como de atención al cliente.

¿Y los metodólatras comportan algún problema en el aprendizaje? Algunos se percatan de las múltiples deficiencias en los proyectos de investigación, pero no pueden asumir las deficiencias de la defectuosa formación profesional desde el pregrado en los egresados, lo que implica un trabajo que supera largamente su capacidad y disposición. En otros casos solo se percatan fragmentariamente del problema. En otros se dificulta discriminar entre la ingenuidad y el dolo. Lo que sí es evidente es su renuencia a la reflexión epistemológica de los problemas que confrontan en el proceso de la investigación.

\section{La caricatura}

Hay una caricatura bastante ácida para graficar este sinsentido: Se cuenta que cierto metodólogo ingenuo, coge una pulga y le ordena saltar, una vez que el bicho lo hace, el investigador hace las mediciones del caso. Coge nuevamente al sujeto experimental y luego de arrancarle una pata le ordena saltar nuevamente. Haciendo uso del instrumento anterior, efectúa la medición correspondiente, repite la operación, hasta que la infortunada pulga (ya sin patas) no obedece la orden del investigador y se mantiene estática, inmutable. Entonces, atendiendo a la hipótesis que se había formulado, nuestro investigador concluye: Se confirma que a medida que se le va quitando las patas a la pulga, ésta va perdiendo la audición hasta que al perder todas las patas, pierde por completo la audición.

La caricatura anterior es análoga a muchas investigaciones en el sistema universitario, y lamentablemente se repite con bastante frecuencia en los egresados no solo del pregrado. Algo parecido ocurre en los postgrados, sin que pueda hacérseles entender la falta de consistencia teórica de las investigaciones que se presentan como tesis para la obtención de las licenciaturas como de los grados académicos posteriores. Este hecho ya fue alertado en diversas ocasiones sin que pueda repararse las deficiencias señaladas al respecto; tampoco las autoridades académicas atinan a ensayar medidas tendientes a reducir este impacto que, además, ya es bastante antiguo y seguramente nos sobrevivirá largamente (Ardila, 1997).

Como consecuencia de este problema no resuelto es que la competencia profesional de los egresados sigue postergándose indefinidamente. Por este mismo motivo, seguramente, se exige la acreditación académica de las instituciones educativas supuestamente superiores, sin embargo los indicadores de tal acreditación no contemplan lo que se propone en este artículo, ya que su tratamiento exige de un trabajo mucho más cuidadoso y a mediano plazo. Pues atender este tipo de carencias académicas lleva mucho más tiempo (Musso, 1970). Una de las consecuencias más notorias de esta carencia epistémica en los trabajos de investigación se observa en la presencia de una falta de liderazgo académico en los egresados, quienes no se encuentran en la capacidad de proponerse soluciones pertinentes a los problemas que confrontan en su actividad cotidiana y, muchas veces, la eluden o se subordinan a otros problemas, formando parte de ellos, como ocurre con las faltas a la 
ética, la corrupción de funcionarios, la defectuosa administración de la justicia, el deterioro en la atención de la salud, la educación, etc.

\section{Las instituciones educativas}

En muy pocas se advierte una cultura organizacional académica permanente, cuyos miembros advierten la necesidad de vigilar el rigor científico en la elaboración de las investigaciones conducentes a la obtención tanto de la licenciatura como de los grados académicos. Lamentablemente, muchas otras la han descuidado parcialmente o por completo. Por eso se advierte gruesas deficiencias en la elaboración, sustentación y aprobación de las mismas. En muchas de ellas se insertan testimonios de su experiencia personal, creencias, supersticiones novelescas, cuando no arengas y agitación políticas; mezcladas con teorías triviales, pre-científicas y fragmentarias de procesos que en la vida diaria se observan en una dinámica continua de interdependencias.

Este tipo de investigaciones que se encuentran claramente en un nivel exploratorio, son convertidas en "descriptivas", incluso "explicativas experimentales" alentados por metodólogos ingenuos o pícaros, quienes muchas veces consiguen hacerlas aprobar recurriendo a otro tipo de artificios ajenos a la actividad académica. Cada vez que nos encontramos con este tipo de casos, deploramos los excesos a los que nos ha conducido la magnificación del empleo de los métodos como única condición para juzgar la pertinencia de las investigaciones que suponemos como científicas.

Lamentablemente estos excesos son bastante comunes y antiguos, seguramente persistirán en el tiempo. Al respecto, Ardila (1997) señala mordazmente que en Harvard, donde él mismo obtuvo el doctorado, también muchos egresados obtienen su doctorado con tesis metodológicamente impecables pero cuya aplicación práctica es nula. Con esto no se pretende justificar las deficiencias de nuestras instituciones, más bien lo que se intenta es sensibilizar a los miembros de la comunidad académica a mostrarnos más vigilantes y a que contribuyan a reducir el impacto de las defraudaciones académicas de este tipo.

Empezar a sensibilizar, no solo a los egresados, involucra también a quienes desarrollan asignaturas de investigación, a los revisores de proyectos de tesis, asesores, jurados de tesis y a los miembros de la comunidad académica para ir reduciendo gradualmente el nefasto impacto de los excesos de la metodolatría. ¿Y las autoridades universitarias? Convendría que se interesaran por estos asuntos, así se percatarían de la inmensa magnitud de la defraudación académica que se advierte en muchísimos de sus egresados. Ya que los recursos que les son asignados son para desempeñar actividades académicas de formación profesional en sus alumnos. Hace algunas décadas atrás, este problema no era tan generalizado como lo es ahora, las pocas universidades de ese tiempo, contaban con los docentes necesarios para conducir todo el proceso de la investigación individualmente, ahora que existen más de 120 universidades, la atención personalizada en esta actividad académica ya es inmanejable, no solo por la masificación de la educación superior cuanto por la inversión de recursos y tiempo que demanda ya son inmanejables.

\section{¿Qué nos induce a incurrir en errores repetidos?}

No nos hemos habituado a testar las teorías que nos orientan en las investigaciones que realizamos (Popper, 1977) y, al no tomar esta precaución elemental, terminamos investigando trivialidades, aconsejados por la ignorancia (Skinner, 1981), sobre todo, en ciencias sociales y humanas donde contamos con diversas teorías, fragmentarias, pre científicas, como ocurre en muchas escuelas de la economía, sociología, educación, psicología etc. (Morín, 1998; Mosterín, 2006, Martel, 2007; Martel-Urbano, 2011).

Estas últimas resultan mucho más necesarias que todas las anteriores, puesto que debieran ayudarnos a elaborar una definición aceptable para la condición humana, aproximarnos a su naturaleza y si esta es singular en cada uno, con mayor razón será necesario intentarlo (Morin, 1999; Mosterín, 2006 ). Estos son algunos de los muchos teóricos quienes nos han advertido la necesidad de orientar nuestros esfuerzos a fin de aproximarnos a una teoría unificada que nos reduzca la mayor cantidad de errores que cometemos al emplear teorías fragmentarias del comportamiento humano.

Disponemos desde hace algún tiempo de teorías unificadas de la psicología que pueden explicarnos el compor- 
tamiento humano: Leontiev (1978), Galperin (1976), Kantor (1990), Pinillos (1983), Ortiz (1994), y recientemente Martel-Urbano (2011), pero no hemos hecho un esfuerzo en aproximarnos a ellas. A esto se debe, en gran medida, que subsistan investigaciones caricaturescas que inducen a investigadores poco experimentados como nuestros egresados, al error. Para esto se requiere que las instituciones educativas alienten a sus miembros a aproximarse a teorías unificadas de la psicología, como ocurre en otras comunidades científicas, cuyos miembros han logrado en poco tiempo reunirse en torno a teorías unificadas, reduciendo el margen de error en sus investigaciones.

La reducción del error, tendrá beneficios prácticos diversos, el adecuado uso de nuestros recursos, racionalizar el empleo de los mismos, evitar gastos superfluos e innecesarios. Para esto se requiere de un esfuerzo en los miembros de nuestra comunidad académica una actitud un poco más racional y menos dogmática, en lugar de seguir protegiendo feudos o monarquías medioevales con súbditos, vasallos y bufones incluidos. Habiéndose iniciado ya la segunda década del este tercer milenio, la propuesta resulta pertinente, ya que el desarrollo de las sociedades dependen, en gran medida de la dinámica que le impriman sus instituciones académicas, educativas, de formación profesional, servicios de salud, etc. Al tratar de enmendar las deficiencias teóricas podemos corregir nuestros errores o podrán hacerlo en el futro próximo algunos lectores sensatos (Popper, 1977).

\section{Todo problema mal planteado, es insoluble. (José Ingenieros, 1937)}

Para destacar la antigüedad con que fue abordado el tema que nos ocupa, se hace referencia a la cita que precede, José Ingenieros ya lo había padecido en su tiempo y hasta la actualidad esta deficiencia continúa vigente en nuestras instituciones educativas superiores, en efecto, esta verdad es cada vez más cierta cada vez que encontramos a muchos licenciados y graduados que no han logrado alcanzar ninguno de estos objetivos: teóricos, prácticos ni personales; en las investigaciones que realizaron para la obtención del grado o la licenciatura. Los objetivos teóricos se refieren al modo cómo el investigador se explica a sí mismo el problema que ha investigado. Esto supone un paso necesario de un estado inicial al inicio de la investigación al estado final una vez concluido. Es de esperar que el investigador adquiera la competencia necesaria para explicarse en mejores condiciones el problema que investigó y pueda explicarnos del modo más explícito posible. Esto involucra encontrar teorías más confiables que expliquen mejor el problema, testar teorías, desechar aquellas defectuosas, hacer epistemología. Lamentablemente, comprobamos que esto no se produce en la mayor parte de los graduados. Este primer objetivo ha sido alcanzado en muy pocos casos.

En cuanto a los objetivos prácticos, a la aplicación de los hallazgos en el contexto educativo para mejorarlo, superando los problemas que se han investigado; esto redundaría en un mayor impulso en el tratamiento de los problemas que aquejan a nuestras instituciones, impulsar las orientaciones más adecuadas para tratar y prevenir los problemas investigados, etc. Este segundo objetivo tampoco ha sido logrado, en la mayor parte de nuestros graduados, las instituciones educativas continúan aquejados de los mismos problemas como al principio.

En cuanto a los objetivos personales, referidos al liderazgo académico que puedan ejercer en sus respectivas instituciones donde laboran, tampoco ocurre en la medida que se requiere, son muy pocos e insuficientes los líderes académicos entre nuestros egresados, frente a la magnitud de los problemas que confrontamos, la mayor parte de ellos resultan frustrados ante la inercia que se advierte en nuestras instituciones. Amedrentados por los grupos de poder y la corrupción optan por el perfil más bajo posible, para no colisionarse con ellos, en otros casos, pasan a engrosar sus filas, o a esperar turno.

\section{A modo de conclusiones}

Para reducir el impacto de este problema, se requiere en primer lugar, recuperar la cultura organizacional académica en nuestras instituciones educativas superiores, actualmente monopolizadas por los grupos de poder. Esto será posible cuando entre en vigencia la nueva ley universitaria que consagre el voto universal y directo en la elección de las autoridades universitarias, habremos dado el primer paso para reducir gradualmente la nefasta influencia de dichos grupos y empezar la construcción de comunidades académicas que permitan la 
formación profesional mejor lograda en nuestros alumnos, evitando la defraudación académica, hoy tan generalizada.

\section{Referencias bibliográficas:}

Ardila, R. (1997). Walden Tres. Barcelona: Biblioteca Ciencias de la Conducta.

Condemarín, M. y otros. (1996) Madurez escolar. Santiago: Andrés Bello.

Daly, M. (1973). Más allá de Dios Padre, hacia una filosofía de la liberación de las mujeres. Nueva York: Harper y Row.

Eco, U. (1986). Cómo se hace una tesis, técnicas, procedimientos de estudio, investigación y escritura. Barcelona: Gedisa.

Eco, U. (1899). El péndulo de Foucault. Bogotá: Bompiani-Lumen.

Feyerabend, P. (1989). Límites de la ciencia. México Paidós.

Feyerabend, P. (2006) Tratado contra el método. Madrid: Tecnos.

Gadamer, H. (1995). El giro hermenéutico. Madrid: Cátedra.

Galperin, P. (1976). Introducción a la psicología. Bs. As: Pablo del Río.

Gonzáles, F. (1996). Problemas epistemológicos de la psicología. La Habana: Academia.

Hernández, R. y otros. (2003). Metodología de la Investigación. México: Mc Graw Hill.

Ingenieros, J.( 1937). Principios de Psicología. Bs. As: Talleres Gráficos de L. J. Rosso.

Kantor, J. (1990). La evolución científica de la psicología. México: Trillas.

Makabe, P. (1987). El cambio epistemológico. Lima: San Marcos.

Martel, H. (2007). Los siete pecados capitales en la Investigación Científica. Recuperado de www.academiaperuana depsicologia.org

Martel-Urbano, V. (2011). La psicología de la actividad psíquica. Lima: EPCUS.

Morin, E. (1998). Los siete saberes necesarios para la educación del futuro. Lima: Derrama Magisterial

Mosterín, J. (2006). Crisis de los paradigmas en el siglo XXI. Lima: Fondo Editorial UPIGV.

Musso, R. (1979). Problemas y mitos metodológicos de la psicología. Bs. As: Psiqué.

Musso, R. (1970). Falacias y mitos metodológicos de la psicología. Bs. As: Psiqué.

Ortiz, P. (1994). El sistema de la personalidad. Lima: Orión.

Popper, K. (1977). Búsqueda sin término. Madrid: Tecnos.

Rotondo, H. (1987). Terapia familiar. Lima: UNMSM.

Sánchez, H. (2008). La Investigación Participativa. Lima: UPRP.

Sierra, R. (1994). Tesis doctorales y trabajos de investigación científica. Madrid: Paraninfo.

Skinner, B. (1981). Conducta verbal. México: Trillas. 